\title{
Molecular Mechanisms and Drug Development in Aquaporin Water Channel Diseases: Water Channel Aquaporin-2 of Kidney Collecting Duct Cells
}

\author{
Kuniaki Takata ${ }^{1, *}$, Yuki Tajika ${ }^{1}$, Toshiyuki Matsuzaki ${ }^{1}$, Takeo Aoki ${ }^{1}$, Takeshi Suzuki ${ }^{1}$, \\ Ablimit Abduxukur ${ }^{1}$, and Haruo Hagiwara ${ }^{1}$ \\ ${ }^{1}$ Department of Anatomy and Cell Biology, Gunma University Graduate School of Medicine, \\ Maebashi, Gunma 371-8511, Japan
}

Received September 29, 2004; Accepted October 13, 2004

\begin{abstract}
Aquaporin-2 (AQP2) is one of the membrane water channel proteins expressed in principal cells of the kidney collecting ducts. In the basal state, AQP2 resides in the storage vesicles localized in the subapical cytoplasm. Upon stimulation with vasopressin, AQP2 is translocated to the apical plasma membrane by the exocytic fusion of the storage vesicles with the apical membrane. This translocation enables the transepithelial reabsorption of water from the lumen to the interstitium via $\mathrm{AQP} 2$ at the apical membrane and $\mathrm{AQP} 3 / \mathrm{AQP} 4$ at the basolateral membrane. AQP2-storage vesicles are distinct from the endoplasmic reticulum, Golgi apparatus, trans-Golgi network, and lysosomes. The early endosomal marker EEA1 is colocalized with some of AQP2 vesicles. Further analyses in Madin-Darby canine kidney (MDCK) cells transfected with AQP2 revealed that subapical Rab11-positive/EEA1-negative smaller vesicles constitute part of the AQP2 storage vesicles for the translocation to the apical membrane. Termination of stimulation results in the retrieval of AQP2 to the larger EEA1-positive early endosomal compartment. AQP2 is then transferred to the subapical storage compartment in a PI3-kinase-dependent manner. GLUT4 is an isoform of glucose transporters whose localization is also regulated by vesicular trafficking induced by insulin stimulation. Comparison of the intracellular localization of AQP2 with GLUT4 suggests distinct regulation of AQP2 trafficking.
\end{abstract}

Keywords: water channel, aquaporin-2, kidney, collecting duct, endosome

\section{Introduction}

Water transport across the cellular membrane is mediated by water channel membrane proteins, aquaporins (AQPs). The water channel was first identified as CHIP28 (channel-like integral protein of $28 \mathrm{kDa}$ ), later termed aquaporin-1 (AQP1), from the human erythrocyte membrane (1). As water transfer is one of the most ubiquitous phenomena of life, aquaporins are widely distributed in all types of life, bacteria, plants, and animals $(2-4)$. In mammalian cells, more than ten isoforms of water channel aquaporins have been identified.

*Corresponding author. FAX: +81-27-220-7900

E-mail: takata@med.gunma-u.ac.jp

\section{Aquaporins in the kidney}

The kidney is an organ specialized for the homeostasis of water in the body. At least 7 isoforms of aquaporins (AQPs 1, 2, 3, 4, 6, 7, and 8) are expressed in the kidney $(4,5)$. Most of them are expressed in the cells of proximal tubules, descending limbs of the Henle's loops, collecting ducts, blood vessels, and renal pelvis. Collecting ducts are the final segment of urine production, where vasopressin-dependent water reabsorption controls the volume of the urine. Water transfer in collecting ducts is mediated by AQP2, AQP3, and AQP4 expressed in principal cells $(4,5)$. In the basal state, AQP2 is localized in cytoplasmic vesicles. It is inserted to the apical plasma membrane by the exocytic process of AQP2-bearing vesicles in response to vasopressin $(5,6)$. This enables the initial step of the reabsorption of 
water: transfer of water through the apical membrane via AQP2. In the basolateral membrane are AQP3 and AQP4, through which water exits from the cell to the interstitium. AQP2 plays a critical role of urine concentration in the kidney, since the mutation of AQP2, either loss of activity or aberrant localization and trafficking, results in diabetes insipidus, the inability to concentrate urine $(6-8)$.

\section{Characterization of intracellular AQP2 storage compartment}

In the basal condition, AQP2 is stored in intracellular vesicles located mostly in the supranuclear/subapical cytoplasm $(5,6,9)$. We characterized the AQP2 vesicles in the principal cells of the rat kidney collecting ducts using semithin frozen sections (10). Double-labeling with organelle-marker proteins revealed that the intracellular AQP2 compartment is distinct from the endoplasmic reticulum, Golgi apparatus, trans-Golgi network (TGN), and lysosome. A portion of AQP2 vesicles are positive for the early endosome marker, early endosome antigen-1 (EEA1), suggesting a possible role of the endosomal system in the intracellular trafficking of AQP2.

To further characterize the intracellular AQP2 compartment, we performed immunohistochemical exami- nation in cultured renal epithelial cells, Madin-Darby canine kidney (MDCK) cells stably transfected with human AQP2 (MDCK-hAQP2 cells) (11). In MDCKhAQP2 cells, AQP2 is localized predominantly in the subapical cytoplasm in the basal state and is translocated to the plasma membrane by increasing the cytoplasmic cyclic AMP level with forskolin (Fig. 1). These features are the same as seen in kidney collecting duct cells, and therefore these AQP2-expressing MDCK cells serve as a good model system for analyzing AQP2 trafficking.

Double-labeling with organelle marker proteins revealed that the intracellular AQP2 compartment is distinct from the endoplasmic reticulum, Golgi apparatus, TGN, and lysosome as is seen in the kidney. In the basal state, AQP2 is localized in close proximity to, but distinct from, EEA1-positive early endosomes. On the other hand, colocalization of AQP2 to Rab11, an apical recycling endosome marker, was seen (11). AQP2 is distinct from the transferrin-loaded basal recycling compartment. These observations indicate that AQP2 is stored in the compartment in/or with close relationship to the endosomal compartment, especially apical recycling endosomes (Fig. 2).

We then compared the intracellular localization of AQP2 with that of glucose transporter GLUT4 (11). GLUT4 is an isoform of facilitated-diffusion-type glucose transporter whose localization in the cell is
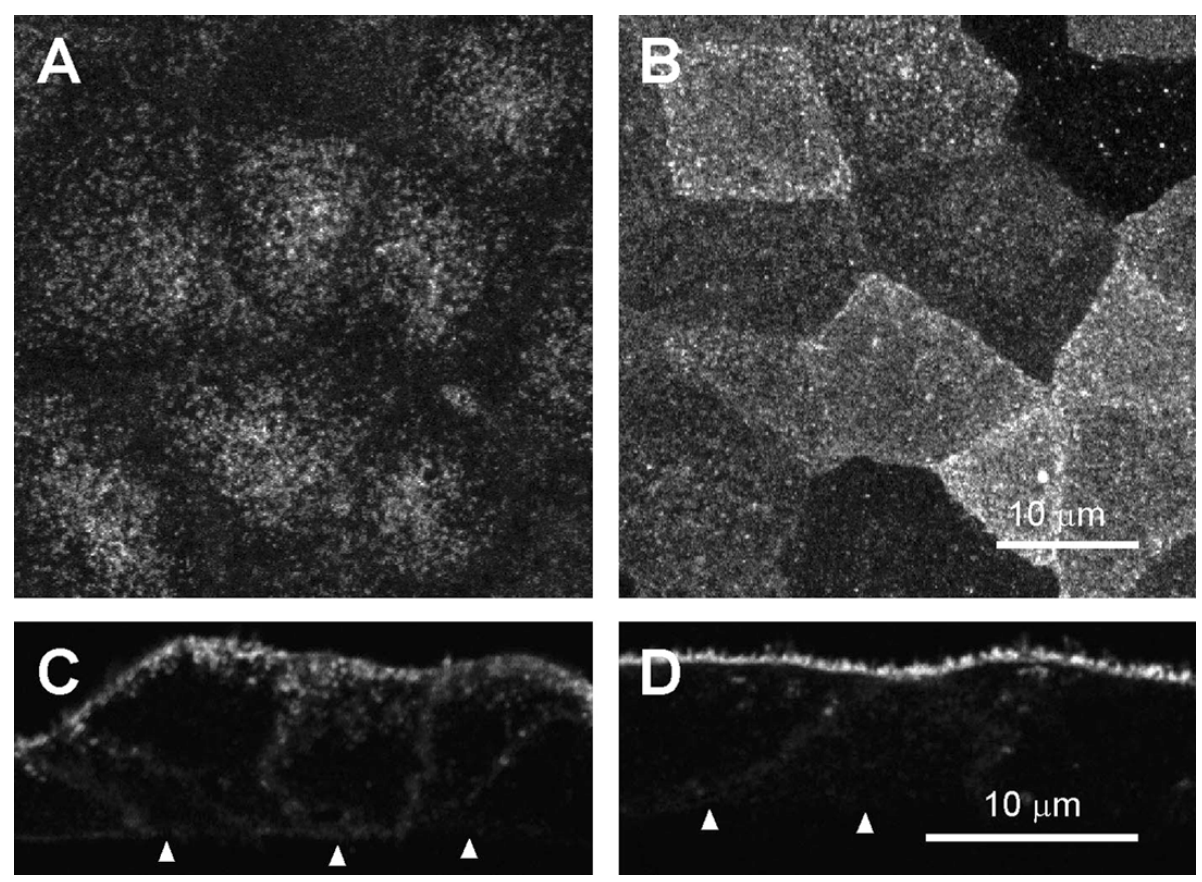

Fig. 1. Translocation of AQP2 in MDCK cells transfected with human AQP2. In the basal state, AQP2 is found in intracellular vesicles mostly localized in the apical cytoplasm $(\mathrm{A}, \mathrm{C})$. Stimulation with forskolin induces the translocation of AQP2 to the apical plasma membrane (B, D). A, B: view from the apical side. C, D: view from the lateral side. Arrowheads indicate basal membrane. 


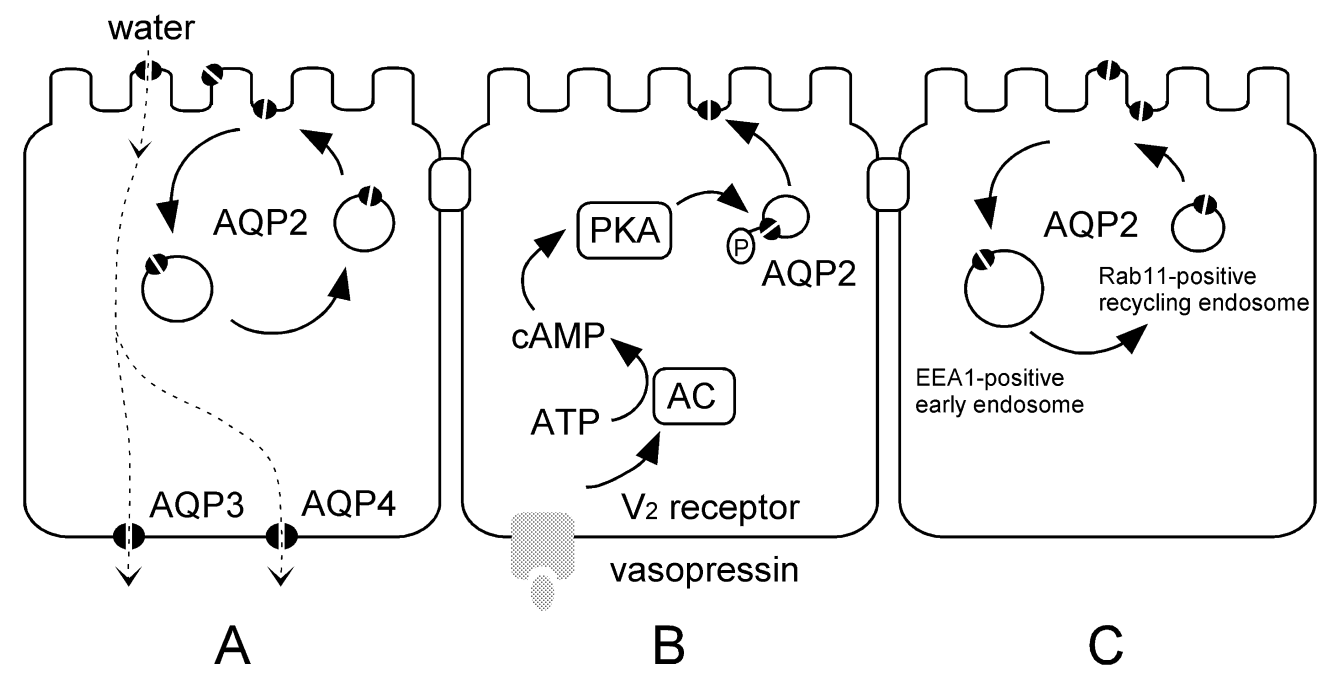

Fig. 2. A schema showing the localization of AQP2 and its trafficking. A: AQP2 is stored in the cytoplasmic vesicles. Once it is translocated to the apical plasma membrane, transepithelial transfer of water occurs via AQP2 at the apical membrane and AQP3/AQP4 at the basolateral membrane. B: Vasopressin binds to the $V_{2}$ receptor, with subsequent activation of adenylate cyclase (AC), and protein kinase A (PKA) induces phosphorylation of AQP2 in the storage vesicles and their exocytic translocation to the plasma membrane. C: Termination of vasopressin stimulation results in the retrieval of plasma membrane AQP2 to the EEA1-positive early endosomes and subsequent transfer to the Rab11-positive storage vesicles.

regulated by insulin (12). As is seen in AQP2, GLUT4 is stored in the intracellular compartment in the basal state. Treatment with insulin induces the translocation of GLUT4 from the intracellular storage compartment to the plasma membrane. Such increase of cell surface GLUT4 is the cellular basis of insulin-stimulated increase of glucose uptake in adipocytes and myocytes. When GLUT4 was transiently expressed in MDCKhAQP2 cells, GLUT4 was also sequestered in intracellular compartments as seen in adipocytes and myocytes, but its localization was distinct from that of AQP2. Although translocation of GULT4 from the intracellular storage compartment to the plasma membrane upon insulin stimulation in adipocytes and myocytes is comparable to that of AQP2 upon vasopressin stimulation in kidney cells, the differential intracellular localization suggests that each of them employs distinct cellular machinery of intracellular storage and regulation of their trafficking. The difference could be attributed to the differential signal transduction machinery for translocation: tyrosine-kinase-mediated system for GULT4 and protein kinase A-mediated system for AQP2.

\section{Translocation of AQP2 to the plasma membrane and its retrieval to the storage compartment}

Forskolin stimulates the translocation of intracellular AQP2 to the apical plasma membrane. Freeze-fracturereplica electron microscopy revealed that an aggregate of intramembranous particles appears at the plasma membrane. These particles were shown to represent AQP2 by the fracture-label technique (13). One of the characteristic features of $\mathrm{AQP} 2$ translocation is that most of the intracellular AQP2 is translocated to the plasma membrane upon stimulation. Only a portion of the intracellular GULT4 is subject to translocation to the plasma membrane, and a large amount of GULT4 is still retained intracellularly in the Golgi and TGN regions even at maximal insulin stimulation. Such a difference may be due to the difference in intracellular storage sites: In AQP2, many more molecules are stored in the "ready-to-go" endosomal compartment near the plasma membrane, whereas in GLUT4, many molecules are stocked in "not-ready-to-go" compartments such as TGN or the Golgi.

When forskolin is washed out, retrieval of cell surface AQP2 to the intracellular compartment commences. Immumolabeling with clathrin showed that AQP2 is concentrated in the clathrin-coated pits (13). Expression of dominant-negative dynamin 1 and 2 revealed that endocytosis is dynamin-dependent (13). Double-labeling with early endosomal marker EEA1 showed the dominance of AQP2/EEA1-double-positive vesicles at the early part of the retrieval process, showing that endocytosed AQP2 first enters the early endosomal compartment (11) (Fig. 2). The AQP2/EEA1-doublepositive vesicles are larger than the original EEA1negative AQP2-storage compartment in diameter and are localized in the supranuclear cytoplasm. Actin 
filaments play an important role in trafficking of the retrieval to the storage compartment since their disruption with cytochalasin $\mathrm{D}$ or latranculin $\mathrm{B}$ resulted in the accumulation of AQP2 in this early endosomal compartment (Y. Tajika and K. Takata, unpublished observation).

AQP2 in the early endosomes is then transferred to the subapical AQP2-storage compartment, a part of which is Rab11-positive (Fig. 2). Wortmannin and LY294002 greatly retarded this transfer, suggesting it is a PI3-kinase-dependent process (11). Since not all the AQP2 vesicles are Rab11-positive in the basal state, identity of the AQP2 storage compartment and its relationship to the apical recycling endosomal compartment remains to be studied in detail.

\section{Involvement of lipid rafts}

The cellular membrane is not a uniform structure. Rather, it consists of distinct domains such as apical and basolateral domains in polarized epithelial cells. Recent studies on the heterogeneity of the membrane structure have been focused on the membrane microdomains called lipid rafts (14). Rafts are detergent-insoluble membrane fractions characterized by the enrichment of cholesterol, glycosphingolipids, and GPI (glycosylphosphatidylinositol)-linked proteins. Caveolae are omega-shaped cell surface indentations rich in membrane proteins caveolins and cholesterol and represent a type of rafts in a broad sense. These rafts and/or caveolae serve as scaffolds for a variety of cellular signaling molecules and hence play critical roles in the intracellular signal transduction and vesicular trafficking.

A significant amount of AQP2 was recovered in the Triton X-100-insoluble fraction in MDCK-hAQP2 cells (T. Aoki and K. Takata, unpublished observation). Little is known, however, about the role of rafts on the translocation and retrieval of AQP2 in kidney cells. In GULT4, possible roles of rafts in the insulin-signal transduction and GLUT4 trafficking have been reported. A small GTP-binding protein TC10 is localized in lipid rafts and its association in rafts and reorganization of cortical F-actin structure were suggested to be involved in the translocation of GLUT4 in adipocytes $(15-17)$. Further analyses on the role of rafts in GLUT4 will shed light on the significance of rafts in the trafficking of membrane molecule trafficking between the plasma membrane and the intracellular storage compartments.

\section{Roles of cytoskeletons in the AQP2 trafficking}

In amphibian urinary bladder and rat kidney inner medullary collecting ducts, vasopressin depolymerizes actin filaments in the apical regions of the granular cells and principal cells, respectively $(18,19)$. These observations suggest that fusion of water channel-bearing vesicles is promoted by the reorganization of cortical actin filaments. Cytochalasin D induced both depolymerization of the F-actin cytoskeleton and AQP2 translocation in primary cultured inner medullary collecting duct cells (20). Inhibition of Rho also induced the depolymerization of the F-actin and AQP2 translocation, whereas expression of constitutively active RhoA induced formation of stress fibers and abolished AQP2 translocation in response to forskolin, indicating that Rho-controlled actin cytoskeleton reorganization regulates the translocation of AQP2 (20).

\section{Perspectives}

Despite the importance of AQP2 in the regulation of urine concentration, the intracellular storage compartment of AQP2 and the regulation of AQP2 trafficking have been poorly understood. Combined molecular and morphological analyses will shed light on this unique feature of this molecule.

\section{Acknowledgments}

We thank Yukiko Tajika-Takahashi and Miki Kusama for assistance. This work was supported in part by Grants-in-Aid for Scientific Research from the Ministry of Education, Culture, Sports, Science, and Technology of Japan and scientific grant from the Kazato Research Foundation.

\section{References}

1 Denker BM, Smith BL, Kuhajda FP, Agre P. Identification, purification, and partial characterization of a novel $\mathrm{Mr} 28,000$ integral membrane protein from erythrocytes and renal tubules. J Biol Chem. 1988;263:15634-15642.

2 Agre P, Sasaki S, Chrispeels MJ. Aquaporins: a family of water channel proteins. Am J Physiol. 1993;265:F461.

3 Matsuzaki T, Tajika Y, Tserentsoodol N, Suzuki T, Aoki T, Hagiwara H, et al. Aquaporins: a water channel family. Anat Sci Int. 2002;77:85-93.

4 Takata K, Matsuzaki T, Tajika Y. Aquaporins: water channel proteins of the cell membrane. Prog Histochem Cytochem. 2004;39:1-83.

5 Nielsen S, Frokiaer J, Marples D, Kwon TH, Agre P, Knepper MA. Aquaporins in the kidney: from molecules to medicine. Physiol Rev. 2002;82:205-244.

6 Sasaki S, Kuwahara M, Yamashita Y, Marumo F. Structure and function of AQP2. Nephrol Dial Transplant. 2000;15 Suppl 6:21-22.

7 Kwon TH, Hager H, Nejsum LN, Andersen ML, Frokiaer J, 
Nielsen S. Physiology and pathophysiology of renal aquaporins. Semin Nephrol. 2001;21:231-238.

8 Knoers NV, Deen PM. Molecular and cellular defects in nephrogenic diabetes insipidus. Pediatr Nephrol. 2001;16:1146-1152.

9 Brown D. The ins and outs of aquaporin-2 trafficking. Am J Physiol Renal Physiol. 2003;284:F893-F901.

10 Tajika Y, Matsuzaki T, Suzuki T, Aoki T, Hagiwara H, Tanaka $\mathrm{S}$, et al. Immunohistochemical characterization of the intracellular pool of water channel aquaporin-2 in the rat kidney. Anat Sci Int. 2002;77:189-195.

11 Tajika Y, Matsuzaki T, Suzuki T, Aoki T, Hagiwara H, Kuwahara M, et al. Aquaporin-2 is retrieved to the apical storage compartment via early endosomes and phosphatidylinositol 3kinase-dependent pathway. Endocrinology. 2004;145:43754383.

12 Malide D, Ramm G, Cushman SW, Slot JW. Immunoelectron microscopic evidence that GLUT4 translocation explains the stimulation of glucose transport in isolated rat white adipose cells. J Cell Sci. 2000;113:4203-4210.

13 Sun TX, Van Hoek A, Huang Y, Bouley R, McLaughlin M, Brown D. Aquaporin-2 localization in clathrin-coated pits: inhibition of endocytosis by dominant-negative dynamin. Am J Physiol Renal Physiol. 2002;282:F998-F1011.
14 Simons K, Ikonen E. Functional rafts in cell membranes. Nature. 1997;387:569-572.

15 Parton RG, Molero JC, Floetenmeyer M, Green KM, James DE. Characterization of a distinct plasma membrane macrodomain in differentiated adipocytes. J Biol Chem. 2002;277:46769-46778.

16 Chamberlain LH, Gould GW. The vesicle- and target-SNARE proteins that mediate Glut4 vesicle fusion are localized in detergent-insoluble lipid rafts present on distinct intracellular membranes. J Biol Chem. 2002;277:49750-49754.

17 Chunqiu Hou J, Pessin JE. Lipid Raft targeting of the TC10 amino terminal domain is responsible for disruption of adipocyte cortical actin. Mol Biol Cell. 2003;14:3578-3591.

18 Ding GH, Franki N, Condeelis J, Hays RM. Vasopressin depolymerizes F-actin in toad bladder epithelial cells. Am J Physiol. 1991;260:C9-C16.

19 Simon H, Gao Y, Franki N, Hays RM. Vasopressin depolymerizes apical $\mathrm{F}$-actin in rat inner medullary collecting duct. Am J Physiol. 1993;265:C757-C762.

20 Klussmann E, Tamma G, Lorenz D, Wiesner B, Maric K, Hofmann F, et al. An inhibitory role of Rho in the vasopressinmediated translocation of aquaporin-2 into cell membranes of renal principal cells. J Biol Chem. 2001;276:20451-20457. 